\title{
Neutral inclusions for diffusive acoustic fields
}

\author{
Lars Kroon and Kenneth Järrendahl
}

Journal Article

\section{Tweet}

N.B.: When citing this work, cite the original article.

Original Publication:

Lars Kroon and Kenneth Järrendahl, Neutral inclusions for diffusive acoustic fields, Journal of Sound and Vibration, 2017. 295, pp.80-89.

http://dx.doi.org/10.1016/j.jsv.2017.02.025

Copyright: Elsevier

http://www.elsevier.com/

Postprint available at: Linköping University Electronic Press

http://urn.kb.se/resolve?urn=urn:nbn:se:liu:diva-136562

(C) $\mathbb{P} \Theta \Theta$

I.U UNNGERSIIET 


\title{
Neutral inclusions for diffusive acoustic fields
}

\author{
Lars Kroon*, Kenneth Järrendahl \\ Department of Physics, Chemistry and Biology, Linköping University, SE-581 83 Linköping, Sweden
}

\begin{abstract}
We predict scattering cancellation in diffusive transport of acoustics waves propagating through multiple scattering media in the stationary limit. This would enable sensing of diffusive sound without disrupting the exterior acoustic field. We present design schemes for making spherical or cylindrical core-shell structures with multiple layers, characterized by homogenous and isotropic diffusion coefficients, neutral to an arbitrary applied multipole field. The double-layered sphere is found to support transparency to two concurrent multipole fields and unique cloaking solutions of arbitrary multipole order. One extra degree of freedom is provided by every layer added to the core-shell structure which may be exploited with our iterative formula for effective diffusivity for cloaking of additional field terms. From this we pass over to the long wavelength limit of ballistic sound and provide formulas for effective mass densities of multi-layered structures in spherical and cylindrical geometries with respect to multipole pressure fields.
\end{abstract}

\section{Introduction}

An acoustic cloak is a shell surrounding a target so that sound incident from any direction passes through and around the cloak, making the cloak and the object undetectable. One cloaking approach is to develop and use metamaterials for guiding sound around the target. This approach prevents wave interaction with the object, and was originally described for electromagnetic waves [1,2], and subsequently extended to acoustic [3, 4, 5] and elastic waves [6, 7], and also to mass diffusion [8]. Another route to cloaking is to also recognize the object properties and construct the cloak to cancel the dominant scattering, rendering the cloak and the object nearly invisible [9]. Using a properly designed isotropic material shell one can achieve cancellation of the monopole and dipole scattered terms. This amounts to making the shell and the target have effective density and compressibility of the surrounding medium [10,11], so that they behave as a neutral acoustic inclusion $[12,13]$. Acoustic scattering cancellation has recent been experimental realized in cylindrical geometry by matching the effective density and compressibility to a water background [14].

In many situations, sound propagation is not ballistic and cannot be described adequately by the acoustic wave equation. For instance, in bubbly water or in other systems containing many randomly distributed scatterers, sound can be thought of as performing a random walk. Effectively, this random walk slows the wave propagation down and scrambles the phase leading to

\footnotetext{
${ }^{*}$ Corresponding author

Email address: lars.kroon@liu.se (Lars Kroon)

Postprint accepted by Journal of Sound and Vibration
} 
diffusive transport [15]. We note that the common approach to describe acoustic wave propagation in bubbly media is to use an effective medium theory that provides an effective phase speed and attenuation while taking into account the void fraction and bubble dynamics [16]. However, if the transport mean free path length in the disordered scattering medium is shorter than the relevant system size and larger than the wavelength of sound, multiple sound scattering can be described by diffusion theory and it is meaningful to talk about a diffusion coefficient.

In this paper, we seek neutrality (or transparency) to multipole fluxes in the stationary limit of diffusive acoustics. This would enable a sensor to detect diffusive sound without disrupting the exterior acoustic field in analogy with the ballistic case [17]. The experimentally determined quantity is the transmitted flux (i.e. the acoustic intensity which is proportional to the square of the detected pressure) which is related to the energy density via Fick's law [18]. In steady-state transport measurements the outcome is determined by the transport mean free path, whereas the diffusion coefficient is obtained from dynamic experiments. In contrast to the acoustic wave equation [4] the time-dependent diffusion equation [8] is not form invariant under a general coordinate transformation so that a diffusive acoustic cloak cannot be designed using a spatial transformation. For stationary diffusion the transformational design approach does apply and gives a spatially dependent diffusivity tensor, which can be difficult to realize in practice. An acoustic bilaminate shell was designed to cancel the scattering of monopole, dipole and quadrupole modes of a compressional (ballistic) wave at the same frequency [19] and there is nothing to prevent this from being achieved also in the diffusive limit. A scheme for calculating the partial scattering amplitudes and the related scattering phases for an arbitrary layered distribution of acoustic material properties was presented in Ref. [20], where the possibility of reducing the acoustic radiation force, which they referred to as suppressing the transport cross section, was investigated by numerical optimization. Ideally, setting all the scattering phases equal to zero yields zero transport cross section with cloaking as a result. However, in the diffusive limit the concept of phase and coherence of a wave is lost altogether.

We present a scheme for making spherical core-shell structures with multiple layers, characterized by isotropic and homogeneous diffusion coefficients, neutral to arbitrary multipole fields applied at infinity. The double-layer shell is found to support transparency to two concurrent multipole fields and unique cloaking solutions of arbitrary multipole order $n \geq 1$. This is an extension of dipole $(n=1)$ cloaks appearing in other contexts [21, 22, 23, 24, 25]. The number of simultaneous transparency solutions, i.e., the two multipole diffusive fields that can penetrate the core without leaving a scattered field outside the shell, is consistent with the result of scattering cancellation of ballistic waves by an acoustic bilaminate shell [19]. We note that thermal transparency and invisibility has been predicted using the scattering cancellation and mantel cloaking concepts for heat diffusion [26]. In this context, our result applies to stationary heat diffusion and constitutes an extension of the dipole heat flux and single-layer shell considered in Ref. [26], which does not support cloaking solutions. One extra degree of freedom is provided by every layer added to the core-shell structure which may be exploited with our iterative formula for effective diffusivity to obtain neutrality to additional field terms. As an example, we give the design recipe for a three-layer shell and show that it supports simultaneous cloaking solutions of two different multipole fields. These results can be adopted to physical phenomena described by the Laplace's equation in spherical symmetry and cylindrical symmetry may be treated with the same methodology. Finally, we pass over to the long wavelength limit of ballistic sound and provide formulas for effective mass densities of multi-layered structures in spherical and cylindrical geometries with respect to multipole pressure fields applied at infinity. 
(a)

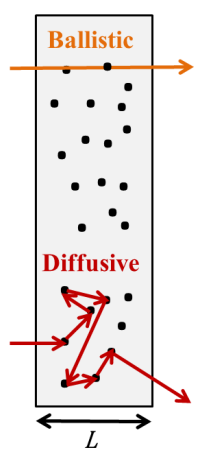

(b)

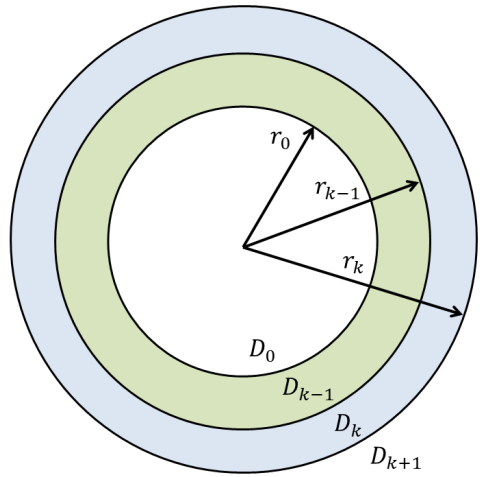

Figure 1: (a) Illustration of ballistic versus diffusive sound paths in a scattering medium of length $L$. (b) Cross section of a spherical core with diffusivity $D_{0}$ and radius $r_{0}$ coated by $k$ concentric laminated layers with diffusion coefficients $D_{1}, \ldots, D_{k}$ and radii $r_{1}, \ldots, r_{k}$ immersed in a medium with diffusivity $D_{k+1}$.

\section{The diffusion approximation}

The description of acoustic waves propagating through strongly scattering media is facilitated by the use of the diffusion approximation, in which all phase information is neglected and the propagation of the multiply scattered waves is treated as a random walk process, see Fig. 1(a). Within this approximation, the dynamic transport is described in terms of the wave diffusion coefficient $D=v_{E} l_{t} / 3$, where $v_{E}$ is the energy velocity, which corresponds to the average local velocity of energy transport in the diffusion process, and $l_{t}$ is the transport mean free path, or the distance the waves must propagate until their direction is randomized. In the absence of absorption the distance between scattering events, or the scattering mean free path $l_{s}$, is related to the transport mean free path as $l_{t}=l_{s} /(1-\langle\cos \theta\rangle)$, where $\langle\cos \theta\rangle$ is the statistical average of the cosine of the scattering angle $\theta$. The two are only equal if the scattering is isotropic. Even in the most strongly scattering medium a coherent, ballistic portion of the transmitted pulse can always be measured if the sample is thin enough. This ballistic pulse consists of the unscattered and/or forward scattered portions of the wave pulse. The scattering mean free path $l_{s}$ can be determined from the attenuation of a ballistic pulse using the definition $I(L)=I_{0} \exp \left(-L / l_{s}\right)$, where $I(L)$ is the transmitted intensity for a sample of thickness $L$ and $I_{0}$ is the incident intensity. The ballistic transmittance $T_{b}=I(L) / I_{0}$ decays exponentially and the remnant effectively vanishes for a thick sample (or can be removed from the total transmittance), whereas the diffusive transmittance may be approximated by $T_{d} \approx l_{t} / L$, decreasing inversely with the sample thickness for large $L$. The description of the pulse shape provides a measure of the diffusion coefficient $D$ of the sound as well as the inelastic absorption time $\tau_{a}$ when compared to the solution of the diffusion equation [27]

$$
\nabla \cdot(D \nabla U)-\frac{\partial U}{\partial t}=0,
$$

where $t$ is time and $U=U_{a} e^{-t / \tau_{a}}$ is the acoustic energy density, including the effect of absorption. The initial conditions and boundary reflections need to be properly treated in the model [27].

The diffusion approximation has been shown to give excellent description of energy transport of multiply scattered ultrasonic waves if the transport mean free path $l_{t} \leq L / 4$, where $L$ is the 
smallest extent of a homogeneously disordered sample [15]. To prevent energy localization the transport mean free path should be larger than the acoustic wavelength, e.g., $l_{t} \geq 5 \lambda$. Under these assumptions we get a possible range of $l_{t} \in[5 \lambda, L / 4]$. The ratio of the upper limit to the lower limit yields a dynamic range for the diffusivity of $D_{\max } / D_{\min }=L / 20 \lambda>1$. For example, for a wavelength of $\lambda=0.5 \mathrm{~mm}$ (corresponding to the ultrasonic frequency $f=3 \mathrm{MHz}$ in water) it requires a sample length of $L=1 \mathrm{dm}$ to obtain one order of magnitude of dynamic range for the diffusivity. Moreover, the absorption mean free path $l_{a}=v_{E} \tau_{a}$ needs to be much larger than the scattering mean free path length, i.e., $l_{a} \gg l_{s}$. This should be kept in mind below when we find ideal cloaking solutions in the extreme limits of zero or infinite diffusivity.

The time-dependent diffusion equation (1) is not form invariant under a general spatial transformation since there is no factor in front of the time derivative $\partial U / \partial t[8]$. This is in contrast to both transformation acoustics [4] and transformation thermodynamics [28]. In the stationary limit, we arrive at the Laplace's equation

$$
\nabla \cdot(D \nabla U)=0,
$$

for which form invariance is recovered and diffusive acoustic fields can be guided around a region by spatial transformations in tune with diffusive light, see the review [29].

\section{Transfer-matrix formalism for neutral inclusions}

We make use a transfer-matrix method to find conditions for multilayered coated spheres to be neutral, or transparent, to multipole diffusive fields applied at infinity. A similar technique was used to study scattering cancellation for elastodynamics [20]. The acoustic energy density in Eq. (2) may be expanded as

$$
U^{(j)}=\sum_{n=0}^{\infty} U_{n}^{(j)}=\sum_{n=0}^{\infty}\left(A_{n}^{(j)} r^{n}+\frac{B_{n}^{(j)}}{r^{n+1}}\right) P_{n}(\cos \theta)
$$

in each diffusivity domain $D_{j}$ for $j=0,1, \ldots, k, k+1$. Here $k \geq 1$ is the number of layers in the shell (see Fig. 1(b)), $r$ is the radius, $\theta$ is the polar angle and $P_{n}$ is the Legendre polynomial of order $n$. The coefficients $A_{n}^{(j)}$ and $B_{n}^{(j)}$ are determined by the boundary conditions. By enforcing continuity of the energy density $U$ and of the normal component of the diffusion flux $\boldsymbol{J}=-D \nabla U$ across the interface $r=r_{j}$, separating the diffusivities $D_{j}$ and $D_{j+1}$, the boundary conditions can be expressed as

$$
\left[\begin{array}{cc}
r_{j}^{2 n+1} & 1 \\
n D_{j} & -(n+1) D_{j} r_{j}^{-(2 n+1)}
\end{array}\right]\left[\begin{array}{c}
A_{n}^{(j)} \\
B_{n}^{(j)}
\end{array}\right]=\left[\begin{array}{cc}
r_{j}^{2 n+1} & 1 \\
n D_{j+1} & -(n+1) D_{j+1} r_{j}^{-(2 n+1)}
\end{array}\right]\left[\begin{array}{c}
A_{n}^{(j+1)} \\
B_{n}^{(j+1)}
\end{array}\right] .
$$

For non-zero $D_{j}$ the conditions can be rewritten as

$$
\left[\begin{array}{c}
A_{n}^{(j)} \\
B_{n}^{(j)}
\end{array}\right]=T_{j}\left[\begin{array}{c}
A_{n}^{(j+1)} \\
B_{n}^{(j+1)}
\end{array}\right], \quad j=0,1, \ldots, k .
$$

The coefficients are related by the transfer matrix

$$
T_{j}=\frac{1}{(2 n+1)\left(1+\Gamma_{j}\right)}\left[\begin{array}{cc}
2 n+1+\Gamma_{j} & 2(n+1) \Gamma_{j} r_{j}^{-(2 n+1)} \\
2 n \Gamma_{j} r_{j}^{2 n+1} & 2 n+1-\Gamma_{j} \\
4 &
\end{array}\right],
$$


where we have defined the mismatch factor

$$
\Gamma_{j}=\frac{D_{j}-D_{j+1}}{D_{j}+D_{j+1}} .
$$

This factor takes values in the range $-1 \leq \Gamma_{j} \leq 1$. The relation between the coefficients interior and exterior to a $k$-layer shell is found by iteration of Eq. (5) as

$$
\left[\begin{array}{c}
A_{n}^{(0)} \\
B_{n}^{(0)}
\end{array}\right]=\prod_{j=0}^{k} T_{j}\left[\begin{array}{c}
A_{n}^{(k+1)} \\
B_{n}^{(k+1)}
\end{array}\right] .
$$

We consider $A_{n}^{(k+1)}$ as given in terms of an applied flux field $\boldsymbol{J}_{n}^{(k+1)}=-D_{k+1} \nabla U_{n}^{(k+1)}$ in the limit $r \rightarrow \infty$, corresponding to a multipole of order $n$ at infinity. The monopole term $\boldsymbol{J}_{0}^{(k+1)}=O\left(r^{-2}\right)$ as $r \rightarrow \infty$, implying that $A_{0}^{(k+1)}$ is an unphysical constant taken to be zero, so we consider $n \geq 1$. Due to the fact that the energy density $U_{n}^{(0)}$ must be finite at the origin, we have $B_{n}^{(0)}=0$. Equating (8) to $B_{n}^{(k+1)}=0$ yields neutrality to each multipole flux $\boldsymbol{J}_{n}^{(k+1)}$ separately. This means that $T_{0} T_{1} \cdots T_{k}$ is an upper triangular matrix. Below, we treat a few-layer shells in some detail and give a formula for effective diffusivity, relating the diffusion coefficients in the general case.

\section{Effective diffusivity with respect to multipole fields}

\subsection{Single-layer shell}

The one-layer shell is defined by letting $r_{2} \rightarrow \infty$ in Fig. 1(b) so that $D_{2}$ becomes the exterior diffusivity. The neutrality condition applied to Eq. (8) with $k=1$ using Eq. (6) becomes

$$
\Gamma_{1}=-\Gamma_{0} \frac{(2 n+1)\left(\frac{r_{0}}{r_{1}}\right)^{2 n+1}}{2 n+1-\Gamma_{0}\left[1-\left(\frac{r_{0}}{r_{1}}\right)^{2 n+1}\right]},
$$

where the allowed values of the mismatch factors $\Gamma_{0}$ and $\Gamma_{1}$ are determined by requiring that the ratio $r_{0} / r_{1}$ is positive and less than unity. Equation (9) is analogous to the electromagnetic transparency condition for Mie scattering on an electrically small coated sphere [9]. By expanding $\Gamma_{0}$ and $\Gamma_{1}$ in Eq. (9) using Eq. (7), we can express the exterior diffusion coefficient as

$$
D_{2}=D_{1}+\frac{(2 n+1) D_{1}\left(D_{0}-D_{1}\right)\left(\frac{r_{0}}{r_{1}}\right)^{2 n+1}}{(2 n+1) D_{1}+n\left(D_{0}-D_{1}\right)\left[1-\left(\frac{r_{0}}{r_{1}}\right)^{2 n+1}\right]} .
$$

The exterior diffusivity $D_{2}$ acts as the effective diffusivity $D_{1}^{\star}$ of the coated inclusion with respect to an arbitrary multipolar field of order $n \geq 1$. The neutrality condition (10) for a uniform field $(n=1)$ is equivalent to the Hashin-Shtrikman formula [30] for the effective diffusivity

$$
D_{1 \mid n=1}^{\star}=D_{1}+\frac{3 D_{1}\left(D_{0}-D_{1}\right) f_{1}}{3 D_{1}+\left(D_{0}-D_{1}\right) f_{0}},
$$

where $f_{1}=1-f_{0}=\left(r_{0} / r_{1}\right)^{3}$ are the filling fractions. In Fig. 2 we plot the mismatch factor (9) as a function of $\Gamma_{0}$ and the corresponding diffusivity contrast $D_{2} / D_{1}$ in Eq. (10) as a function of $D_{0} / D_{1}$ for neutrality to dipole $(n=1)$, quadrupole $(n=2)$ and octupole $(n=3)$ flux fields. The thickness ratio is $r_{0} / r_{1}=0.75$. The only common solution is the trivial one with $D_{0}=D_{1}=D_{2}$. 

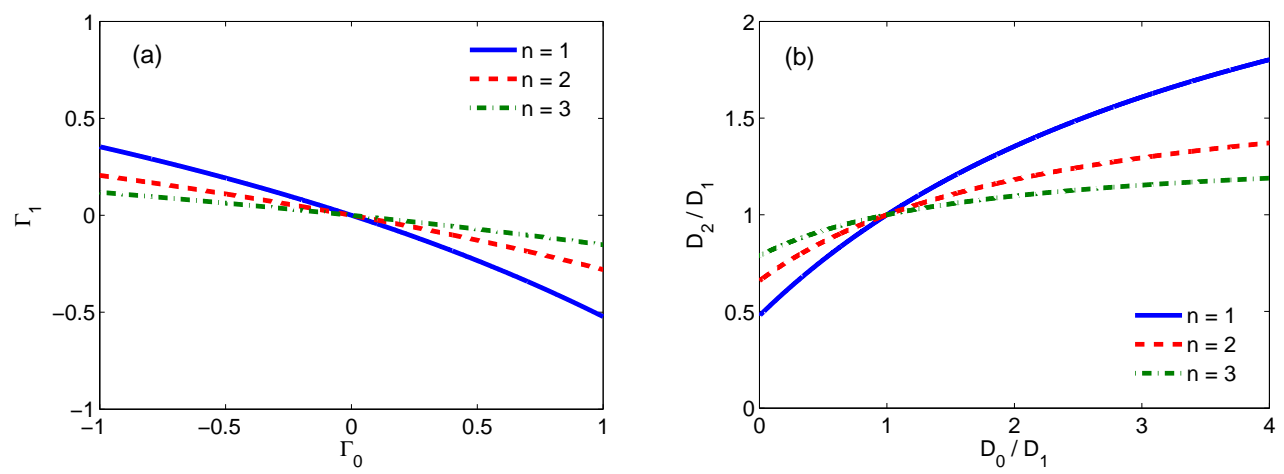

Figure 2: (a) The mismatch factor $\Gamma_{1}$ in Eq. (9) as a function of $\Gamma_{0}$ and (b) the diffusivity ratio $D_{2} / D_{1}$ in Eq. (10) as a function of $D_{0} / D_{1}$ for neutrality to dipole $(n=1)$, quadrupole $(n=2)$ and octupole $(n=3)$ flux fields. The thickness ratio is $r_{0} / r_{1}=0.75$.

\subsection{Double-layer shell}

For the two-layer shell in Fig. 1(b) the neutrality condition applied to Eq. (8) with $k=2$ using Eq. (6) is

$$
\frac{2(n+1) \Gamma_{2}}{2 n+1+\Gamma_{2}}=-\frac{\left[\Gamma_{1}+\Gamma_{0} \frac{2 n+1+\Gamma_{1}}{2 n+1-\Gamma_{0}}\left(\frac{r_{0}}{r_{1}}\right)^{2 n+1}\right]\left(\frac{r_{1}}{r_{2}}\right)^{2 n+1}}{\left[\frac{2 n+1-\Gamma_{1}}{2(n+1)}+\frac{2 n \Gamma_{0} \Gamma_{1}}{2 n+1-\Gamma_{0}}\left(\frac{r_{0}}{r_{1}}\right)^{2 n+1}\right]},
$$

where the allowed values of the mismatch factors $\Gamma_{0}, \Gamma_{1}$ and $\Gamma_{2}$ are determined by requiring that the ratios $r_{0} / r_{1}$ and $r_{1} / r_{2}$ are positive and less than unity. For a given geometry the mismatch factors $\Gamma_{1}$ and $\Gamma_{2}$ can be uniquely determined from Eq. (12) with $n=1,2$ as functions of $\Gamma_{0}$ [31]. By expanding $\Gamma_{1}$ and $\Gamma_{2}$ in Eq. (12) using Eq. (7), we find the diffusivity relation

$$
D_{3}=D_{2}+\frac{(2 n+1) D_{2}\left(D_{1}^{\star}-D_{2}\right)\left(\frac{r_{1}}{r_{2}}\right)^{2 n+1}}{(2 n+1) D_{2}+n\left(D_{1}^{\star}-D_{2}\right)\left[1-\left(\frac{r_{1}}{r_{2}}\right)^{2 n+1}\right]},
$$

where

$$
D_{1}^{\star}=D_{0} \frac{\left(1-\Gamma_{0}\right)}{\left(1+\Gamma_{0}\right)} \frac{\left[1+\frac{2(n+1) \Gamma_{0}}{2 n+1-\Gamma_{0}}\left(\frac{r_{0}}{r_{1}}\right)^{2 n+1}\right]}{\left[1-\frac{2 n \Gamma_{0}}{2 n+1-\Gamma_{0}}\left(\frac{r_{0}}{r_{1}}\right)^{2 n+1}\right]}
$$

is the effective diffusivity of the single-layer sphere (10), expressed in terms of the mismatch factor $\Gamma_{0}$, the diffusivity $D_{0}$ and the ratio $r_{0} / r_{1}$. In Fig. 3 we plot the diffusivity contrast $D_{3} / D_{2}$ in Eq. (13) for neutrality to dipole $(n=1)$, quadrupole $(n=2)$ and octupole $(n=3)$ flux fields as functions of $\Gamma_{0}$ for two characteristic values of $D_{0} / D_{2}$ using thickness ratios $r_{0} / r_{1}=0.75$ and $r_{1} / r_{2}=0.8$. When $D_{0} / D_{2}<1$ (Fig. 3(a)) the curves (13) for two arbitrary $n$ intersect in the range $-1<\Gamma_{0}<0$, corresponding to simultaneous solutions at a specific diffusivity ratio $D_{3} / D_{2}>1$. The intersection points are quite close to each other in parameter space and they can easily be found analytically by solving the quadratic equation (13) for $D_{2} \geq 0$. Likewise when $D_{0} / D_{2}>1$ 

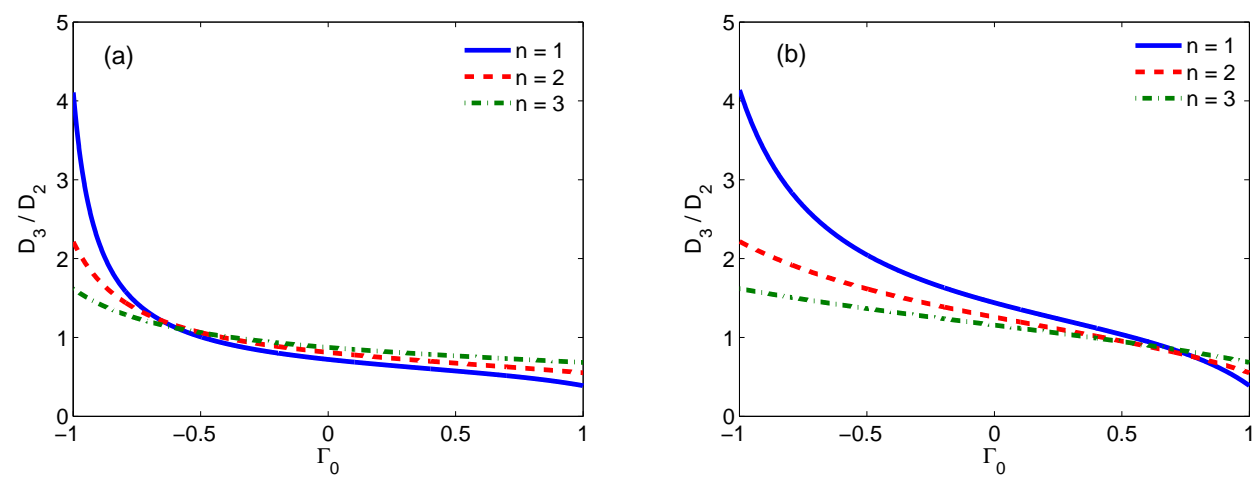

Figure 3: The diffusivity ratios $D_{3} / D_{2}$ yielding neutrality to dipole $(n=1)$, quadrupole $(n=2)$ and octupole $(n=3)$ flux fields as functions of $\Gamma_{0}$ in the case (a) $D_{0} / D_{2}=0.5$ and (b) $D_{0} / D_{2}=2$. The thickness ratios are $r_{0} / r_{1}=0.75$ and $r_{1} / r_{2}=0.8$.

(Fig. 3(b)) the curves (13) for two different $n$ intersect in the range $0<\Gamma_{0}<1$, corresponding to simultaneous solutions at a specific diffusivity ratio $D_{3} / D_{2}<1$. If $D_{0} / D_{2}=1$ all curves will intersect each other at $\Gamma_{0}=0$, corresponding to the trivial solution $D_{0}=D_{1}=D_{2}=D_{3}$.

A two-layer acoustic shell surrounding a spherical object was designed in Ref. [19] by numerical optimization to reduce the scattering of monopole $(n=0)$, dipole $(n=1)$ and quadrupole $(n=2)$ modes of a compressional wave at the same frequency. The number of simultaneous transparency solutions for diffusive acoustic waves, i.e., the pair of multipole flux fields that can penetrate the core (with diffusivity $D_{0}$ different from that of the ambient medium $D_{3}$ ) without leaving a scattered field outside the shell, is consistent with that of the scattering cancellation of ballistic waves by the two-layer acoustic shell [19] since the monopole term is zero in our case. In the limit $D_{1} \rightarrow 0\left(\Gamma_{0} \rightarrow 1\right)$, the diffusivity relation (13) reduces to

$$
\frac{D_{3}}{D_{2}}=\frac{1-\left(\frac{r_{1}}{r_{2}}\right)^{2 n+1}}{1+\frac{n}{n+1}\left(\frac{r_{1}}{r_{2}}\right)^{2 n+1}} .
$$

This gives perfect screening of the core and thus defines a cloaking solution for the applied multipole field of order $n \geq 1$, depending on the ratios $D_{3} / D_{2}$ and $r_{1} / r_{2}$. This formula is an extension of dipole cloaks $(n=1)$ considered earlier in different areas of physics [21, 22, 23, 24, 25]. In the limit $D_{1} \rightarrow \infty\left(\Gamma_{0} \rightarrow-1\right)$, we obtain

$$
\frac{D_{3}}{D_{2}}=\frac{1+\frac{n+1}{n}\left(\frac{r_{1}}{r_{2}}\right)^{2 n+1}}{1-\left(\frac{r_{1}}{r_{2}}\right)^{2 n+1}},
$$

which is another cloaking solution of order $n \geq 1$. In comparison to the two-layer acoustic cloak [19] for ballistic waves these limits would correspond to an inner layer with mass density $\rho_{1}$ approaching infinity and zero, respectively, reducing the degree of freedom to strictly cancel the scattering of two modes at the same frequency. In Fig. 4(a) and 4(b) we plot the diffusivity ratio $D_{3} / D_{2}$ from (15) and (16), respectively, as functions of the thickness ratio $r_{1} / r_{2}$ for cloaking 

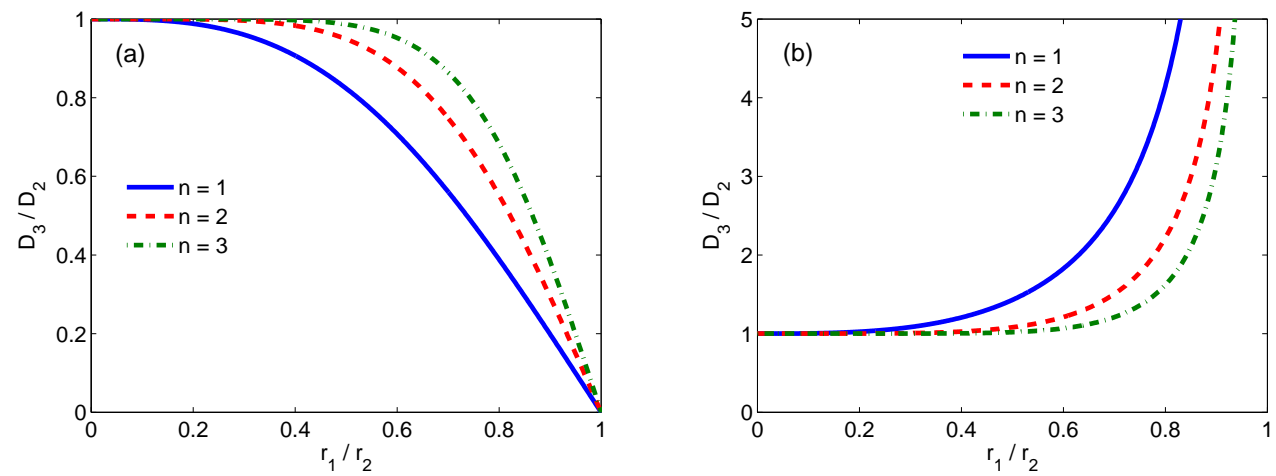

Figure 4: The diffusivity ratios $D_{3} / D_{2}$ as a function of $r_{1} / r_{2}$ for cloaking of dipole $(n=1)$, quadrupole $(n=2)$ and octupole $\left(n=3\right.$ ) flux fields in the case of (a) $D_{1}=0$ and (b) in the limit $D_{1} \rightarrow \infty$.

of dipole, quadrupole and octupole fields. In the limit $r_{1} / r_{2} \rightarrow 1$ the ratio (15) tends to zero while the ratio (16) approaches infinity. The cloaking solutions can, of course, also be found directly from the neutrality condition (12). The limit $D_{1} \rightarrow \infty$ corresponds to $\Gamma_{0}=-\Gamma_{1}=-1$ in which case (12) reduces to $2 n \Gamma_{2} /\left(2 n+1+\Gamma_{2}\right)=-\left(r_{1} / r_{2}\right)^{2 n+1}$, being identical to the expression (16). In the case $\Gamma_{0}=-\Gamma_{1}=1$, corresponding to $D_{1}=0$, the neutrality condition (12) reduces to $2(n+1) \Gamma_{2} /\left(2 n+1+\Gamma_{2}\right)=\left(r_{1} / r_{2}\right)^{2 n+1}$, which is equivalent to the solution (15). The double-layer shell thus supports unique cloaking solutions for every multipole diffusive field of order $n \geq 1$, that is, there are no concurrent solutions since the curves in Fig. 4 never intersect each other.

\subsection{Multi-layer shells}

We find that the neutrality condition for a $k$-layered sphere can be calculated iteratively by setting the exterior diffusivity $D_{k+1}$ equal to its effective diffusivity (with respect to a multipole field of order $n \geq 1$ )

$$
D_{k}^{\star}=D_{k}+\frac{(2 n+1) D_{k}\left(D_{k-1}^{\star}-D_{k}\right)\left(\frac{r_{k-1}}{r_{k}}\right)^{2 n+1}}{(2 n+1) D_{k}+n\left(D_{k-1}^{\star}-D_{k}\right)\left[1-\left(\frac{r_{k-1}}{r_{k}}\right)^{2 n+1}\right]}, \quad k \geq 1,
$$

where $D_{k}$ is the diffusivity of the $k$ th layer, $r_{k-1} / r_{k}$ is the corresponding thickness ratio and $D_{k-1}^{\star}$ is the effective diffusivity of the $(k-1)$-layered sphere, taking $D_{0}^{\star}$ to be the core diffusivity $D_{0}$. As an example, we consider the three-layer shell $(k=3)$ defined by confining $D_{3}$ to the layer $r_{2}<r<r_{3}$ in Fig. 1(b) and letting $D_{4}$ be the exterior diffusivity. In the case of zero diffusivity of the innermost layer, $D_{1}=0$, we obtain

$$
\frac{D_{4}}{D_{3}}=1+\frac{(2 n+1)\left\{\frac{D_{2}}{D_{3}}\left[\frac{1-\left(\frac{r_{1}}{r_{2}}\right)^{2 n+1}}{1+\frac{n}{n+1}\left(\frac{r_{1}}{r_{2}}\right)^{2 n+1}}\right]-1\right\}\left(\frac{r_{2}}{r_{3}}\right)^{2 n+1}}{(2 n+1)+n\left\{\frac{D_{2}}{D_{3}}\left[\frac{1-\left(\frac{r_{1}}{r_{2}}\right)^{2 n+1}}{1+\frac{n}{n+1}\left(\frac{r_{1}}{r_{2}}\right)^{2 n+1}}\right]-1\right\}\left[1-\left(\frac{r_{2}}{r_{3}}\right)^{2 n+1}\right]}
$$


using $D_{3}^{\star}=D_{4}$ and $D_{2}^{\star}=D_{3}$ in Eq. (15) for the effective diffusivities. In Fig. 5(a) the diffusivity ratio $D_{4} / D_{3}$ is plotted as a function of $D_{2} / D_{3}$ for cloaking of dipole $(n=1)$, quadrupole $(n=2)$ and octupole $(n=3)$ fields, respectively. The thickness ratios are $r_{1} / r_{2}=0.8$ and $r_{2} / r_{3}=0.8$. At the intersection points there exist simultaneous cloaking solutions of two different orders. The cloaking solutions (18) can also be expressed in terms of mismatch factors as

$$
\frac{2(n+1) \Gamma_{3}}{2 n+1+\Gamma_{3}}=-\frac{\left[\frac{2(n+1) \Gamma_{2}}{2 n+1+\Gamma_{2}}-\left(\frac{r_{1}}{r_{2}}\right)^{2 n+1}\right]\left(\frac{r_{2}}{r_{3}}\right)^{2 n+1}}{\left[\frac{2 n+1-\Gamma_{2}}{2 n+1+\Gamma_{2}}-\frac{2 n \Gamma_{2}}{2 n+1+\Gamma_{2}}\left(\frac{r_{1}}{r_{2}}\right)^{2 n+1}\right]},
$$

which is the neutrality condition obtained from Eq. (8) with $k=3$ using Eq. (6) in the case $\Gamma_{0}=-\Gamma_{1}=1$. For a given geometry the relation (19) defines cloaking solutions of order $n$ with two degrees of freedom, constituted by the mismatch factors $\Gamma_{2}$ and $\Gamma_{3}$. In Fig. 5(b) we plot $\Gamma_{3}$ in Eq. (19) with the geometry $r_{1} / r_{2}=r_{2} / r_{3}=0.8$ as a function of $\Gamma_{2}$ for cloaking of dipole, quadrupole and octupole fields, respectively. Curves corresponding to different orders intersect each other once in the ranges $0<\Gamma_{2}<1$ and $-1<\Gamma_{3}<0$. The intersection points can be found analytically by solving (19) for the order pairs. One extra degree of freedom is provided by every layer added to the structure which may be exploited for cloaking of additional multipole fields. In general, we observe that the diffusivity ratio $D_{k+1} / D_{k}>1$ if $D_{k-1} / D_{k}>1$ or $D_{k+1} / D_{k}<1$ if $D_{k-1} / D_{k}<1$ for the simultaneous solutions. This means that the diffusion coefficients in the layered structure need to alternate between high and low values with respect to the unit value. These values can be calculated iteratively with the formula (17) for a multi-layered sphere, which is an extension of neutral coated inclusions $[13,32]$ to multipolar fields. For an assemblage of coated spheres the applied field must, however, be uniform to assure neutrality. In this context we mention that the scattering cancellation method has been applied to cases of acoustic waves interacting with non-spherical elastic objects and collections of closely packed objects using an elastic core and spherical fluid layers to represent the objects as effective spherical targets [33].
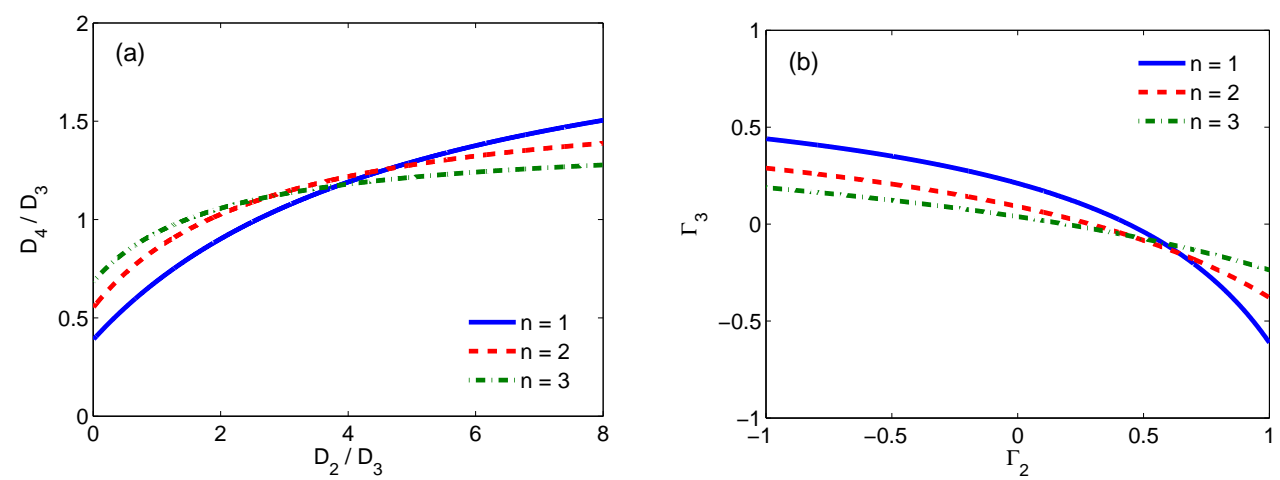

Figure 5: (a) The diffusivity ratio $D_{4} / D_{3}$ in Eq. (18) as a function of $D_{2} / D_{3}$ and (b) the corresponding mismatch factor $\Gamma_{3}$ in Eq. (19) as a function of $\Gamma_{2}$ for cloaking of dipole $(n=1)$, quadrupole $(n=2)$ and octupole $(n=3)$ fields, respectively. The thickness ratios are $r_{1} / r_{2}=0.8$ and $r_{2} / r_{3}=0.8$. 


\section{The long wavelength limit}

The results for diffusive acoustics can be adopted to the transport of ballistic waves in the long wavelength limit, i.e., for components of the transmitted field that travel straight through the medium without scattering out of the forward direction, see Fig. 1(a). Small amplitude disturbances in a compressible fluid are governed by the acoustic wave equation

$$
\nabla \cdot\left(\rho^{-1} \nabla p\right)=\kappa \frac{\partial^{2} p}{\partial t^{2}}
$$

where $p$ is the pressure, $\rho$ is the mass density, $\kappa$ is the compressibility and $t$ is time. The group velocity $v_{g}=\nabla_{\mathbf{k}} \omega$, as the gradient of the angular frequency $\omega$ with respect to the wave vector $\mathbf{k}$, is usually well behaved and corresponds to the velocity of energy transport by ballistic waves. In the long wavelength limit $(\omega \rightarrow 0)$ the time-harmonic fields in Eq. (20) reach the steady state

$$
\nabla \cdot\left(\rho^{-1} \nabla p\right)=0,
$$

which is of the same form as Eq. (2), replacing the diffusion coefficient by the reciprocal mass density $\left(D \leftrightarrow \rho^{-1}\right)$ and the acoustic energy density by the pressure $(U \leftrightarrow p)$. This form occurs in many situations of physics and cloaking, including static magnetic cloaks [21], stationary thermal cloaks [22, 23], direct current electric cloaks [24] and diffusive light cloaks [25]. Continuity at the boundaries between spherical layers of different mass densities requires matching of the pressure and the normal velocity, and hence the radial acceleration $-\rho^{-1} \partial p / \partial r$. This implies that the neutrality condition for a $k$-layered sphere can be calculated iteratively by setting the exterior reciprocal density $\rho_{k+1}^{-1}$ equal to the effective value (in analogy with Eq. (17))

$$
\rho_{k}^{-1 \star}=\rho_{k}^{-1}+\frac{(2 n+1) \rho_{k}^{-1}\left(\rho_{k-1}^{-1}{ }^{\star}-\rho_{k}^{-1}\right)\left(\frac{r_{k-1}}{r_{k}}\right)^{2 n+1}}{(2 n+1) \rho_{k}^{-1}+n\left(\rho_{k-1}^{-1}{ }^{\star}-\rho_{k}^{-1}\right)\left[1-\left(\frac{r_{k-1}}{r_{k}}\right)^{2 n+1}\right]}, \quad k \geq 1,
$$

where $\rho_{k}^{-1}$ is the reciprocal density of the $k$ th layer, $r_{k-1} / r_{k}$ is the corresponding thickness ratio and $\rho_{k-1}^{-1}{ }^{\star}$ is the effective reciprocal density of the $(k-1)$-layered sphere, using $\rho_{0}^{-1^{\star}}=\rho_{0}^{-1}$ for the core. Equation (22) is readily rewritten as a recursion formula for the mass densities

$$
\rho_{k}^{\star}=\rho_{k}+\frac{(2 n+1) \rho_{k}\left(\rho_{k-1}^{\star}-\rho_{k}\right)\left(\frac{r_{k-1}}{r_{k}}\right)^{2 n+1}}{(2 n+1) \rho_{k}+(n+1)\left(\rho_{k-1}^{\star}-\rho_{k}\right)\left[1-\left(\frac{r_{k-1}}{r_{k}}\right)^{2 n+1}\right]}, \quad k \geq 1 .
$$

The mass density itself is not a transport quantity. In the dipole case $(n=1)$ the effective reciprocal mass density (22) realizes the Hashin-Shtrikman bound (11) for a two-phase fluid, whereas the effective mass density (23) is in agreement with Berryman's formula [34]

$$
\rho_{1 \mid n=1}^{\star}=\rho_{1}+\frac{3 \rho_{1}\left(\rho_{0}-\rho_{1}\right) f_{1}}{3 \rho_{1}+2\left(\rho_{0}-\rho_{1}\right) f_{0}},
$$

where $f_{1}=1-f_{0}=\left(r_{0} / r_{1}\right)^{3}$ are the filling fractions. The latter has been utilized to design spherical coated inclusions that are neutral to uniform fields [10]. Matching of the effective density with the ambient medium shows significant reduction in scattering also beyond the quasistatic approximation [35]. We can find density distributions that are simultaneously transparent to multipole pressure fields in the long wavelength limit of ballistic sound either by setting the exterior density $\rho_{k+1}$ equal to the effective density (23) or by matching the reciprocals via (22). 


\section{The cylindrical case}

It is possible to derive analogous theoretical results for other canonical geometries. In the cylindrical case, for instance, following similar steps it may be shown that the neutrality condition for a two-dimensional (2D) disc with $k$ annuli can be calculated iteratively by setting the exterior diffusivity $D_{k+1}$ equal to its effective value (with respect to a multipole field of order $n \geq 1$ )

$$
D_{k}^{\star}=D_{k}+\frac{2 D_{k}\left(D_{k-1}^{\star}-D_{k}\right)\left(\frac{r_{k-1}}{r_{k}}\right)^{2 n}}{2 D_{k}+\left(D_{k-1}^{\star}-D_{k}\right)\left[1-\left(\frac{r_{k-1}}{r_{k}}\right)^{2 n}\right]}, \quad k \geq 1,
$$

where $D_{k}$ is the diffusivity of the $k$ th annulus, $r_{k-1} / r_{k}$ is the corresponding thickness ratio and $D_{k-1}^{\star}$ is the effective diffusivity of the disc with $(k-1)$ annuli, taking $D_{0}^{\star}=D_{0}$ for the central disc. This is the cylindrical analogue of Eq. (17). For example, applying the formula (25) to a disc with two annuli (cf. Fig. 1) with $D_{1}=0$ yields the cloaking recipe

$$
\frac{D_{3}}{D_{2}}=\frac{1-\left(\frac{r_{1}}{r_{2}}\right)^{2 n}}{1+\left(\frac{r_{1}}{r_{2}}\right)^{2 n}}
$$

for diffusive fields of multipole order $n \geq 1$. This is the analogue of Eq. (15) in cylindrical symmetry. We also note that the $2 \mathrm{D}$ analogue of the effective mass density (23) is

$$
\rho_{k}^{\star}=\rho_{k}+\frac{2 \rho_{k}\left(\rho_{k-1}^{\star}-\rho_{k}\right)\left(\frac{r_{k-1}}{r_{k}}\right)^{2 n}}{2 \rho_{k}+\left(\rho_{k-1}^{\star}-\rho_{k}\right)\left[1-\left(\frac{r_{k-1}}{r_{k}}\right)^{2 n}\right]}, \quad k \geq 1,
$$

for pressure fields of multipole order $n \geq 1$ in the long wavelength limit of ballistic sound. This equation is obtained by formally identifying the diffusion coefficients with the reciprocal mass densities $\left(D \leftrightarrow \rho^{-1}\right)$ in Eq. (25) and then rewriting it in terms of the mass densities. In contrast to the 3D spherical case the effective equations for the reciprocal mass densities (cf. Eq. (25)) and the mass densities (27) are identical in form. This result seems to reflect the fact the acoustic wave equation is isomorphic to single polarization Maxwell equations in 2D [3]. Finally, we mention that acoustic transparency at low frequencies has been predicted in a cylindrical system with internal structure when the effective density matches that of the ambient fluid [36].

\section{Conclusion}

In conclusion, we studied cloaking of multipole acoustic fields in multiple scattering media by approximating strong scattering by diffusion in the stationary limit. We presented an iterative scheme for making spherical core-shell structures with multiple layers, characterized by isotropic and homogenous diffusion coefficients, neutral to arbitrary applied multipole fields from which cloaking solutions for every multipole order was found. The two-layer cloaking formulas are extensions of the dipole case considered earlier in different areas of physics [21, 22, 23, 24, 25]. One extra degree of freedom is provided by every layer added to the core-shell structure which can be exploited with our iterative formula for effective diffusivity to obtain neutrality to additional field terms. The results suggest that $k$-layer shells support simultaneous cloaking solutions of $k-1$ different multipole fields. We expect that near-cloaking in diffusive acoustics can be experimentally 
realized with broadband operation and that the conceivable object can be macroscopic in size, like for diffusive light cloaks [25]. The results can be adopted to physical phenomena described by the Laplace's equation in spherical symmetry, and cylindrical symmetry may be treated with the same methodology. Finally, we passed over to the long wavelength limit of ballistic sound and provided formulas for effective mass densities of multi-layered structures in spherical and cylindrical geometries with respect to multipole pressure fields. One may envision that this design can be useful for stealth technology, non-invasive sensing in complex environments and for probing disordered media.

\section{References}

[1] J. B. Pendry, D. Schurig, D. R. Smith, Controlling electromagnetic fields, Science 312 (2006) 1780-1782.

[2] D. Schurig, J. J. Mock, B. J. Justice, S. A. Cummer, J. B. Pendry, A. F. Starr, D. R. Smith, Metamaterial electromagnetic cloak at microwave frequencies, Science 314 (2006) 977-980.

[3] S. A. Cummer, D. Schurig, One path to acoustic cloaking, New Journal of Physics 9 (2007) 45.

[4] H. Chen, C. T. Chan, Acoustic cloaking in three dimensions using acoustic metamaterials, Applied Physics Letters 91 (2007) 183518.

[5] S. A. Cummer, B.-I. Popa, D. Schurig, D. R. Smith, J. Pendry, M. Rahm, A. Starr, Scattering theory derivation of a 3d acoustic cloaking shell, Physical Review Letters 100 (2008) 024301.

[6] G. W. Milton, M. Briane, J. R. Willis, On cloaking for elasticity and physical equations with a transformation invariant form, New Journal of Physics 8 (2006) 248.

[7] A. N. Norris, Acoustic cloaking theory, Proceedings of the Royal Society of London A: Mathematical, Physical and Engineering Sciences 464 (2008) 2411-2434.

[8] S. Guenneau, T. M. Puvirajesinghe, Fick's second law transformed: one path to cloaking in mass diffusion, Journal of The Royal Society Interface 10 (2013) 83.

[9] A. Alù, N. Engheta, Achieving transparency with plasmonic and metamaterial coatings, Physical Review E 72 (2005) 016623.

[10] X. Zhou, G. Hu, Acoustic wave transparency for a multilayered sphere with acoustic metamaterials, Physical Review E 75 (2007) 046606.

[11] M. D. Guild, A. Alù, M. R. Haberman, Cancellation of acoustic scattering from an elastic sphere, The Journal of the Acoustical Society of America 129 (2011) 1355-1365.

[12] E. H. Mansfield, Neutral holes in plane sheet-reinforced holes which are elastically equivalent to the uncut sheet, The Quarterly Journal of Mechanics and Applied Mathematics 6 (1953) 370-378.

[13] G. W. Milton, The Theory of Composites, Cambridge University Press, Cambridge, England, 2002.

[14] C. A. Rohde, T. P. Martin, M. D. Guild, C. N. Layman, C. J. Naify, M. Nicholas, A. L. Thangawng, D. C. Calvo, G. J. Orris, Experimental demonstration of underwater acoustic scattering cancellation, Scientific Reports 5 (2015) 13175.

[15] J. Page, I. Jones, H. Schriemer, M. Cowan, P. Sheng, D. Weitz, Diffusive transport of acoustic waves in strongly scattering media, Physica B 263264 (1999) 37-39.

[16] K. W. Commander, A. Prosperetti, Linear pressure waves in bubbly liquids: Comparison between theory and experiments, The Journal of the Acoustical Society of America 85 (1989) 732-746.

[17] M. D. Guild, A. Alù, M. R. Haberman, Cloaking of an acoustic sensor using scattering cancellation, Applied Physics Letters 105 (2014) 023510.

[18] H. Kuttruff, Room Acoustics, Spon Press, Abingdon, Oxon, United Kingdom, 2009.

[19] M. D. Guild, M. R. Haberman, A. Alù, Plasmonic-type acoustic cloak made of a bilaminate shell, Physical Review B 86 (2012) 104302.

[20] P. T. Bowen, Y. A. Urzhumov, Three forms of omnidirectional acoustic invisibility engineered using fast elastodynamic transfer-matrix method, Journal of Optics 18 (2016) 044025.

[21] F. Gömöry, M. Solovyov, J. Šouc, C. Navau, J. Prat-Camps, A. Sanchez, Experimental realization of a magnetic cloak, Science 335 (2012) 1466-1468.

[22] H. Xu, X. Shi, F. Gao, H. Sun, B. Zhang, Ultrathin three-dimensional thermal cloak, Physical Review Letters 112 (2014) 054301

[23] T. Han, X. Bai, D. Gao, J. T. L. Thong, B. Li, C.-W. Qiu, Experimental demonstration of a bilayer thermal cloak, Physical Review Letters 112 (2014) 054302.

[24] L. Kroon, Anticonductors: cloaks for uniform static currents, in: 8th International Congress 
on Advanced Electromagnetic Materials in Microwaves and Optics (Metamaterials), 2014, pp. $175-177$. doi:10.1109/MetaMaterials.2014.6948636.

[25] R. Schittny, M. Kadic, T. Bückmann, M. Wegener, Invisibility cloaking in a diffusive light scattering medium, Science 345 (2014) 427-429.

[26] M. Farhat, P.-Y. Chen, H. Bagci, C. Amra, S. Guenneau, A. Alù, Thermal invisibility based on scattering cancellation and mantle cloaking, Scientific Reports 5 (2015) 9876.

[27] J. H. Page, H. P. Schriemer, A. E. Bailey, D. A. Weitz, Experimental test of the diffusion approximation for multiply scattered sound, Physical Review E 52 (1995) 3106-3114.

[28] S. Guenneau, C. Amra, D. Veynante, Transformation thermodynamics: cloaking and concentrating heat flux, Optics Express 20 (2012) 8207-8218.

[29] R. Schittny, A. Niemeyer, F. Mayer, A. Naber, M. Kadic, M. Wegener, Invisibility cloaking in light-scattering media, Laser \& Photonics Reviews 10 (2016) 382-408.

[30] Z. Hashin, S. Shtrikman, A variational approach to the theory of the effective magnetic permeability of multiphase materials, Journal of Applied Physics 33 (1962) 3125-3131.

[31] X. Wang, P. Schiavone, A neutral multi-coated sphere under non-uniform electric field in conductivity, Zeitschrift für angewandte Mathematik und Physik 64 (2013) 895-903.

[32] X. He, L. Wu, Thermal transparency with the concept of neutral inclusion, Physical Review E 88 (2013) 033201.

[33] M. D. Guild, A. J. Hicks, M. R. Haberman, A. Al, P. S. Wilson, Acoustic scattering cancellation of irregular objects surrounded by spherical layers in the resonant regime, Journal of Applied Physics 118 (2015) 164903.

[34] J. G. Berryman, Long wavelength propagation in composite elastic media I. Spherical inclusions, The Journal of the Acoustical Society of America 68 (1980) 1809-1819.

[35] X. Zhou, G. Hu, T. Lu, Elastic wave transparency of a solid sphere coated with metamaterials, Physical Review B 77 (2008) 024101.

[36] A. S. Titovich, A. N. Norris, Acoustic scattering from an infinitely long cylindrical shell with an internal mass attached by multiple axisymmetrically distributed stiffeners, Journal of Sound and Vibration 338 (2015) $134-153$. 